\title{
Alternative treatment for secretory diarrhea revealed in a new class of CFTR inhibitors
}

\author{
Qais Al-Awqati \\ Department of Medicine, Columbia University, New York, New York, USA \\ J. Clin. Invest. 110:1599-1601 (2002). doi:10.1172/JCI200217301.
}

Diarrhea remains the number one killer of children less than 5 years of age throughout the world. A large number of organisms are responsible including viruses (rotaviruses), bacteria (Escherichia coli and cholera vibrios) and parasites. It may not be obvious to most people but we are living during the sixth pandemic of cholera in modern memory. This pandemic started in the Celebes Islands in the late 1950's, marched around the globe in less than two decades and now every continent has had reported cases. Unlike previous pandemics, the causative organism is Vibrio El Tor not the classical $V$. cholerae. Studies prompted by this most recent pandemic have contributed an immense increase in our understanding of the mechanism underlying diarrhea. Robert Koch, in his original description of cholera seen among pilgrims in the Egyptian city of El Tor, found ulceration of the intestinal mucosa and postulated that the cause of diarrhea was an increased leak of plasma into the lumen. More recent research has now shown that the Vibrio exotoxin induces intestinal secretion, a process that was previously unknown (1). Cholera exotoxin binds to ganglioside GM1 on the apical surface and after internalization causes an

\footnotetext{
Address correspondence to: Qais Al-Awqati, Department of Medicine and Physiology, Columbia University College Of Physicians \& Surgeons, 630 West 168th Street, New York, New York 10032, USA.

Phone: (212) 305-3512; Fax: (212) 305-3475;

E-mail: qa1@columbia.edu.

Conflict of interest: The author has declared

that no conflict of interest exists.

Nonstandard abbreviations used: cystic

fibrosis transmembrane conductance

regulator (CFTR); $\mathrm{NaK2Cl} \mathrm{cotransporter}$

(NKCC); yellow fluorescent protein (YFP).
}

increase in cellular cyclic AMP levels (2). The toxin ADP-ribosylates adenylyl cyclase resulting in a permanent activation of the enzyme (3). The increased level of cyclic AMP activates chloride secretion by the intestinal mucosa (4). Exotoxins of other bacteria, such as E. coli, activate guanylate cyclase and the resultant cyclic GMP also induces secretion (5). The mainstay of therapy in secretory diarrheas has been the justly celebrated oral rehydration therapy, which singlehandedly has been responsible for reducing the mortality of children by more than half (6). A report in this issue of the JCI by Ma, Verkman, and their colleagues describes the development of an inhibitor of chloride secretion that might serve as a useful addition to our armamentarium (7). A second report in this issue, by Musch and colleagues has resurrected the Koch idea by showing that immune-mediated diarrhea causes an increased leakiness of the intestinal epithelium (8).

\section{Epithelial ion secretion in cholera and cystic fibrosis}

Intestinal electrolyte secretion occurs by a two-step process (Figure 1). Chloride enters the intestinal epithelial cell from the basolateral surface by an electroneutral carrier that translocates $\mathrm{Na}^{+}, \mathrm{K}^{+}$, and two $\mathrm{Cl}^{-}$ions raising the electrochemical potential for $\mathrm{Cl}^{-}$in the cell. The apical membrane contains a cyclic AMP activated $\mathrm{Cl}^{-}$channel which, when open, causes the secretion of $\mathrm{Cl}^{-}$into the lumen (9). Electroneutrality is maintained by secretion of $\mathrm{Na}^{+}$, largely through the tight junctions. The small intestine is quite water-permeable and the accumulated salts therefore lead to the withdrawal of water from the circulation resulting in an iso-osmotic fluid diarrhea. Fluid secretion by the same mechanism occurs in other epithelia including the tracheo-bronchial mucosa, pancreatic ducts, and male genital tract.

During study of the pathophysiology of cystic fibrosis, it was found that the chloride channel of the apical membrane of lung and intestinal epithelia could not be opened by cyclic AMP-dependent protein kinase (10). When the gene was identified it turned out to be a member of the ATP binding cassette proteins similar to the multidrug resistance protein, a family well known to transport a variety of solutes (even proteins) in all organisms, but none were previously known to be ion channels (11). However, definitive evidence was provided that this protein, named CFTR (cystic fibrosis transmembrane conductance regulator), is indeed a chloride channel. Like another member of this family, the sulfonylurea receptor, CFTR was found to regulate the properties of several other ion channels (12).

CFTR is a chloride channel with a conductance that does not depend on the membrane potential. The study of the biophysics of ion channel conductance has benefited from the availability of inhibitors or activators as tools to study the detailed mechanism of permeation of ions through these proteins. Until now there had been no specific inhibitor or activator of CFTR. Most of the inhibitors used had potencies in the submillimolar range, clearly well above what is needed for making significant biophysical conclusions, let alone thinking of their use in clinical practice. Ma, Verkman, and their colleagues, a group well-known for their technical versatility and innovation, have provided a remarkable solution to this problem. 
a

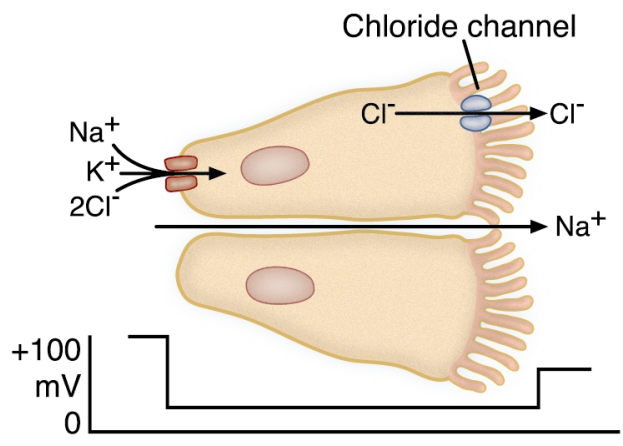

c

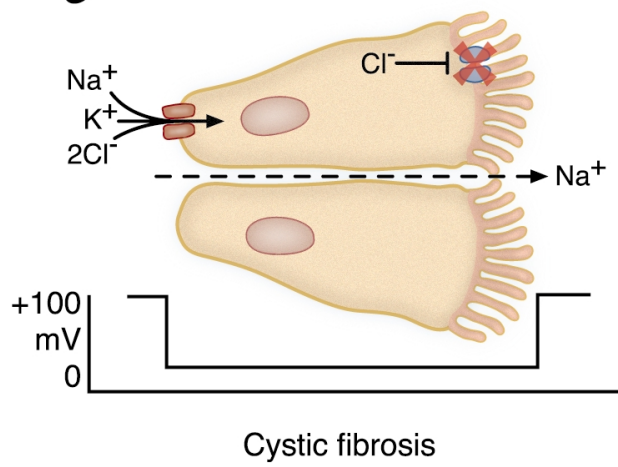

b

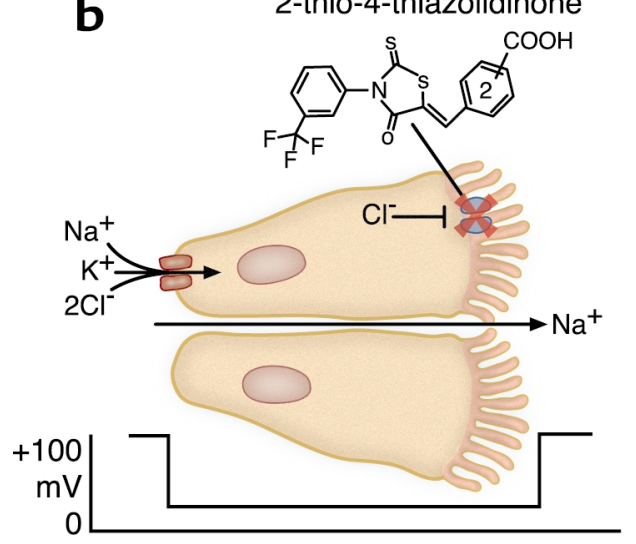

d

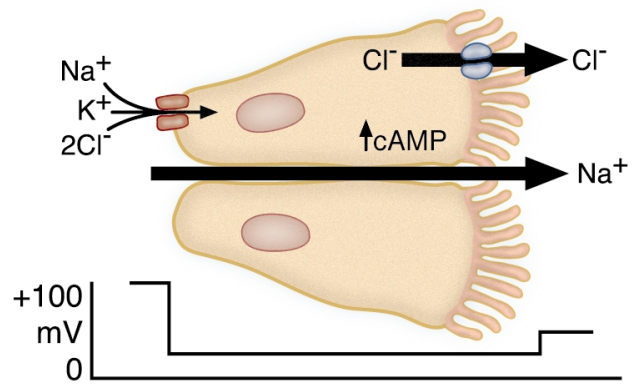

Secretory diarrhea
Figure 1

Epithelial ion secretion: (a) $\mathrm{Na}^{+}$, $\mathrm{K}^{+}$, and $2 \mathrm{Cl}^{-}$ions are transported into the cell by the $\mathrm{NaK} 2 \mathrm{Cl}$ cotransporter (NKCC). $\mathrm{Na}^{+}$and $\mathrm{K}^{+}$ are recycled across the basolateral surface by the NaK-ATPase and a $\mathrm{K}^{+}$channel, respectively. The activity of intracellular $\mathrm{Cl}^{-}$is above electrochemical equilibrium. The apical membrane contains the CFTR, a cyclic AMP-activated $\mathrm{Cl}^{-}$channel. When opened, CFTR-mediated $\mathrm{Cl}^{-}$ secretion results in hyperpolarization of the epithelium leading to secretion of $\mathrm{Na}^{+}$in between the cells. (b) The CFTR is inhibited by thiazolidinone derivatives. (c) In cystic fibrosis epithelia, the CFTR is either not delivered to the apical surface or cannot be opened by cyclic AMP. (d) In secretory diarrhea, an increase in cyclic AMP or cyclic GMP opens the CFTR and leads to increased $\mathrm{Cl}^{-}$secretion.

by Ken Beauchamp J. Clin. Invest.

Development of new drugs is (correctly, I believe) thought to be the province of the pharmaceutical industry. Yet this group courageously tackled this problem by first developing an ingenious assay. They engineered a mutant yellow fluorescent protein (YFP) whose fluorescence is halide dependent (the quantum yield of the fluorophore is reduced on binding to iodide). Iodide permeates through the CFTR; hence the fluorescence intensity of normal epithelial cells that stably express the YFP is quenched when the cells are treated with cyclic AMP. Using these cells in a high-throughput screen of thousands of drug-like small molecules they came up with a structure that seemed to inhibit the CFTR. They identified an organic compound - thiazolidinone - that potently inhibited CFTRmediated chloride transport. Analogs of this compound were potent in the submicromolar range. The inhibition was not due to a reduction in cyclic AMP. As expected, this compound also inhibited cholera toxin-induced fluid secretion. In another report, the same group used a similar approach to identify compounds that opened the CFTR chloride channel with potency in the micromolar range (13).

\section{A new treatment \\ for secretory diarrhea?}

Will this drug (or a more potent derivative) be useful in the treatment of secretory diarrhea? Dehydration remains the major cause of morbidity and mortality in cholera and other diarrheas and any reduction in the fluid loss would certainly be helpful. While there are other drugs that inhibit intestinal secretion, most do so by blocking the $\mathrm{NaK} 2 \mathrm{Cl}$ cotransporter (NKCC), and their use would result in more sodium wasting but in this case by the kidneys (14). Mature kidney tubules do not exhibit chloride secretion hence the use of the new chloride channel-blockers confers some specificity allowing these new compounds to be used in the treatment of diarrhea with impunity. Antibiotics shorten the duration of diarrhea in cholera and other secretory diarrheas. However, many of the infections, including cholera, are self-limiting and the aim of medical therapy remains the prevention and treatment of severe dehydration making oral rehydration the standard of care.

Initially, intravenous saline therapy was used; indeed intravenous infusions were first invented for the treatment of cholera in London in the late 19th Century. Based on physiological principles, it was recently found that oral rehydration was possible, a discovery that is one of the triumphs of modern biomedical research (15). Although cyclic AMP causes secretion it has a minor (if any) effect on the process of $\mathrm{Na}^{+}$absorption. In particular the major process of sodium adsorption whereby sodium transport is directly coupled to glucose and amino acid uptake is intact (3). Provision of sodium with glucose (or substances like rice powder that could be converted to glucose) affords a cheap and easy to administer therapy that prevents dehydration. Fans of medical folklore immediately recognized that boiled rice water has always been the mainstay of treatment of infantile diarrhea in the third world. Similarly, one can also make a strong case for chicken soup, which has a high peptide content that could be hydrolyzed 
to amino acids and afford large amounts of cotransported solutes for sodium absorption. Before images of sage grandmothers with infinite primal wisdom carry one away, it should be emphasized that only mixtures that contain the appropriate proportions of salts and cotransported solutes reduced the diarrhea-related mortality of infants in poor countries. These discoveries were capitalized on to make the ubiquitous Gatorade and other "sports drinks" in wealthy countries.

As has been the experience with many new drugs, new indications are likely to arise as physicians learn to use thiazolidinone drugs. The authors have already thought of another potential target: polycystic kidney disease. It has been determined that the cyst-lining epithelia secrete fluid by a CFTR-mediated channel (16). One potential indication for the new drug is to prevent cyst expansion in polycystic kidney disease.

One of the most interesting possibilities for the use of thiazolidinone derivatives is in the study of cystic fibrosis in humans. The pathophysiology of cystic fibrosis is still obscure (17). Especially problematic has been the colonization and infection of the lung first by Staphylococcus aureus followed by Pseudomonas aeruginosa. Mutant mice lacking the CFTR do not develop significant pulmonary disease, perhaps not surprisingly given that infectious diseases are ecological perturbations that require intimate hostpathogen interaction of a high degree of specificity. Research on this problem has suffered from the fact that by the time the subjects have well developed cystic fibrosis, chronic inflammation has usually set in with consequent destruction of the lung. The use of human subjects treated with this drug might uncover the early mechanism of bacterial colonization unaffected by chronic lung disease.

1. Carpenter, C.C., Sack, R.B., Feeley, J.C., and Steenberg, R.W. 1968. Site and characteristics of electrolyte loss and effect of intraluminal glucose in experimental canine cholera. J. Clin. Invest. 47:1210-1220.

2. Kimberg, D.V., Field, M., Johnson, J., Henderson, A., and Gershon, E. 1971. Stimulation of intestinal mucosal adenyl cyclase by cholera enterotoxin and prostaglandins. J. Clin. Invest. 50:1218-1230.

3. Moss, J., and Vaughan, M. 1977. Choleragen activation of solubilized adenylate cyclase: requirement for GTP and protein activator for demonstration of enzymatic activity. Proc. Natl. Acad. Sci. USA. 74:4396-4400.

4. Field, M., Fromm, D., Al-Awqati, Q., and Greenough, W.B., III. 1972. Effect of cholera enterotoxin on ion transport across isolated ileal mucosa. J. Clin. Invest. 51:796-804.

5. Field, M., Graf, L.H., Jr., Laird, W.J., and Smith, P.L. 1978. Heat-stable enterotoxin of Escherichid coli: in vitro effects on guanylate cyclase activity, cyclic GMP concentration, and ion transport in small intestine. Proc. Natl. Acad. Sci. USA 75:2800-2804

6. Victora, C.G., Bryce, J., Fontaine, O., and Monasch,
R. 2000. Reducing deaths from diarrhoea through oral rehydration therapy. Bulletin of the World Health Organization. 78:1246-1255.

7. Ma, T., et al. 2002. Thiazolidinone CFTR inhibitor identified by high-throughput screening blocks cholera toxin-induced intestinal fluid secretion. J. Clin. Invest. 110:1651-1658. doi:10.1172/JCI200216112.

8. Musch, M.W., et al. 2002. T cell activation causes diarrhea by increasing intestinal permeability and inhibiting epithelial $\mathrm{Na}^{+} / \mathrm{K}^{+}$-ATPase. J. Clin. Invest. 110:1739-1747. doi:10.1172/JCI200215695.

9. Frizzell, R.A., Field, M., and Schultz, S.G. 1979 Sodium-coupled chloride transport by epithelial tissues. Am. J. Physiol. 236:F1-F8.

10. Anderson, M.P., Sheppard, D.N., Berger, H.A., and Welsh, M.J. 1992. Chloride channels in the apical membrane of normal and cystic fibrosis airway and intestinal epithelia. Am. J. Physiol. 263:L1-L14.

11. Riordan, J.R., et al. 1989. Identification of the cystic fibrosis gene: cloning and characterization of complementary DNA. Science. 245:1066-1073.

12. Schwiebert, E.M., Benos, D.J., Egan, M.E., Stutts, M.J., and Guggino, W.B. 1999. CFTR is a conductance regulator as well as a chloride channel. Physiol. Rev. 79:S145-S166.

13. Ma, T., et al. 2002. High-affinity activators of cystic fibrosis transmembrane conductance regulator (CFTR) chloride conductance identified by high-throughput screening. J. Biol. Chem. 277:37235-37241.

14. Al-Awqati, Q., Field, M., and Greenough, W.B., III. 1974. Reversal of cyclic AMP-mediated intestinal secretion by ethacrynic acid. J. Clin. Invest. 53:687-692.

15. da Cunha Ferreira, R.M., and Cash, R.A. 1990 History of the development of oral rehydration therapy. Clin. Ther. 12(Suppl. A):2-11.

16. Davidow, C.J., Maser, R.L., Rome, L.A., Calvet, J.P. and Grantham, J.J. 1996. The cystic fibrosis transmembrane conductance regulator mediates transepithelial fluid secretion by human autosomal dominant polycystic kidney disease epithelium in vitro. Kidney. Int. 50:208-218.

17. Milewski, J.M., and Frizzell, R.A. 1999. Role of CFTR in airway disease. Physiol Rev. 79:S215-S255. 